\title{
Research on Virtual Power Plant Optimal Scheduling Based on TOU Electricity Price
}

\author{
Dunnan Liu ${ }^{1}$, Yuan Gao ${ }^{1 *}$, Tingting Zhang ${ }^{1}$, Zhixin Dong ${ }^{1}$, Haiyu Huang ${ }^{2}$, Chunming Wang ${ }^{2}$ and Shaolian $\mathrm{Xi}^{2}$ \\ ${ }^{1}$ State Key Laboratory of Alternate Electrical Power System With Renewable Energy Sources(North China Electric Power University), \\ Changping District, Beijing 102206, China \\ ${ }^{2}$ Central China Electric Power Dispatching and Control Sub-center of State Grid, Wuhan 430061, China
}

\begin{abstract}
The power supply in many provinces is in short supply during peak load period, and the largescale entry of new energy into the grid brings new problems to the safety and economic operation of the grid. Under the mechanism of time-of-use electricity price, the virtual power plant participates in the grid dispatching by integrating distributed new energy and demand-side resources, which can effectively participate in the peak load clipping and valley filling of the grid and suppress the load fluctuation of the grid. This paper builds a VPP economic optimization scheduling model based on TOU electricity price, and uses particle swarm optimization algorithm to get the output of each energy source of VPP. The simulation results show that the model is reasonable and economical.
\end{abstract}

\section{Introduction}

In recent years, renewable energy such as wind power and photovoltaic power has been connected to the grid on a large scale, which has reduced the power generation space of thermal power units. Combined with its intermittency and fluctuation characteristics, the peak regulation pressure of the power grid has increased. The peak regulation of existing thermal power units cannot fully meet the demand, so it is necessary to excavate the demand-side resources. Virtual power plant (VPP) aggregates energy storage, controllable load (DG), distributed power supply and other resources, and its participation in the market can effectively relieve the pressure of grid peak regulation [1-2].

At present, the research of domestic and foreign scholars on virtual power plant mainly focuses on the planning and market transaction of virtual power plant. Literature [3-4] builds a robust bidding model for virtual power plants to participate in day-ahead market, and the model can minimize the operating cost of virtual power plants. Literature [5] proposed the orderly charging and discharging model of electric vehicles under the time-ofuse price. Literature [6] proposed the energy and reserve bidding model of virtual power plant taking into account the thermal inertia of the building. Literature [7-8] proposed a robust optimal bidding model for virtual power plants, and solved it alternately through dual decomposition. Literature [9-10] studied the clearance mechanism of Shanghai Virtual Power Plant participating in peak regulation and gave the clearance process, but did not establish the corresponding mathematical model.
The above literatures rarely consider the influence of TOU electricity price on VPP. For VPP, taking TOU electricity price into consideration when participating in peak load cutting and valley filling can effectively reduce the cost of power generation and enable VPP to obtain greater economic benefits. In this paper, a VPP operation strategy based on TOU electricity price is designed, and a VPP economic optimization scheduling model based on TOU electricity price is constructed with the goal of maximizing revenue in each period. Particle swarm optimization algorithm is adopted to get the output of each energy source of VPP. The simulation results show that the model is reasonable and economical.

\section{Operation Strategy of Virtual Power Plant Based on TOU Electricity Price}

\subsection{Influence of TOU Electricity Price on Virtual Power Plant Operation}

A typical generation side VPP consists of an uncontrollable DG, a controllable power supply and an energy storage system. After the implementation of TOU electricity price, when the power generation cost of the controllable power source is higher than the TOU electricity price, the power generation cost of VPP will be increased. VPP can arrange the power generation plan of the controllable power source according to the TOU electricity price to reduce the power generation cost. The energy storage system has the characteristic of transferring electricity. After the implementation of TOU electricity price, VPP can transfer the low-price electricity of the distribution network to sell it when the

Corresponding author: gaoyuanhd@163.com 
distribution network has a high price through the energy storage system, and obtain benefits. The implementation of TOU will encourage VPP to adjust the power generation plan to reduce the cost of power generation and participate in peak load cutting and valley filling of distribution network to obtain better benefits.

\subsection{Operation Mode of Virtual Power Plant Based on TOU Electricity Price}

The operation mode of VPP includes the following steps:

(1) The next day uncontrollable DG output was predicted by VPP, and the uncontrollable DG output curve every 15 min on the next day was obtained.

(2) Optimize next-day VPP output plan and declare next-day VPP output plan by comprehensively considering factors such as uncontrollable DG forecast output curve, time-of-use electricity price and power generation costs of various distributed energy sources.

(3) The dispatching department shall confirm the declared output plan of VPP and arrange the output of VPP and other generating units in the region.

(4) After considering the operation strategy of VPP based on TOU electricity price, VPP carries out economic optimization scheduling and obtains the realtime optimization scheduling results of the next day.

\subsection{Operation Strategy of Virtual Power Plant Based on TOU Electricity Price}

(1) Uncontrollable DG operation strategy

Wind power generation and photovoltaic power generation are connected to the grid as new energy power, and VPP gives priority to use within its ability range.

(2) Operation strategy of controllable power supply

On the basis of preferential utilization of new energy power, the operation strategy of gas turbine considering its own generation cost and TOU price is as follows: the generation cost is higher than the electricity price sold, and the gas turbine stops; The cost of electricity generation is lower than the price of electricity sold, and the gas turbine generates electricity; When uncontrollable DG generates more power and the energy storage system cannot be completely absorbed, the gas turbine generates less power according to the situation.

(3) Operation strategy of energy storage system

Output deviation refers to the difference between the uncontrollable DG planned output and the actual output in the next day, and the uncontrollable DG output deviation is random in size. The output deviation is compensated through the joint operation of pumped storage energy and energy storage battery, so that the actual VPP output can track the planned output.

1. Output deviation is greater than 0 , the uncontrollable DG generation is insufficient, the VPP energy storage system generates electricity, and when insufficient, the electricity purchased from distribution can meet the VPP output plan: the deviation is small, the energy storage battery generates electricity; When the deviation is large and exceeds the minimum output of the turbine, the pumped storage energy and the energy storage battery will jointly operate for power generation.

2. Output deviation is less than 0 , uncontrollable DG generation exceeds, and VPP energy storage system stores energy. If the energy storage power is less than the deviation power, gas turbine output will be reduced to meet the VPP output plan: small deviation, energy storage battery storage; Big deviation. When the deviation exceeds the pumping power of the pump, the pumped storage energy and the energy storage battery are combined for energy storage.

3. Pumped storage energy can be purchased for energy storage during the valley period of TOU electricity price and sold during the peak period of TOU electricity price, which can obtain economic benefits and play a certain role of peak clipping and valley filling.

\section{Optimal dispatching model of virtual power plant based on TOU price}

\subsection{Objective function}

Taking the maximum economic benefits in each period of VPP as the goal, considering the operation management cost of each distributed energy source, fuel cost of gas turbine and penalty cost of deviation from output plan, the objective function is:

$$
\begin{aligned}
& \max F_{k}^{V P P}=\sum_{k}\left[I_{k}^{S E}-C_{k}^{O M}-C_{k}^{F}-C_{k}^{P E}\right] \\
& I_{k}^{S E}=P_{1}^{k}\left(g_{k}^{W H}+g_{k}^{P V}+g_{k}^{G T}+g_{k}^{t u r}+g_{k}^{d i s}-g_{k}^{p u}-g_{k}^{c h}\right) \\
& C_{k}^{O H}=\mathrm{K}_{O M}^{W} \mathrm{~g}_{k}^{W}+\mathrm{K}_{O H}^{P V} \mathrm{~g}_{k}^{P V}+\mathrm{K}_{O, H}^{G T} \mathrm{~g}_{k}^{G T}+\mathrm{K}_{O M}^{\text {pumped }} \\
& \left(\mathrm{g}_{k}^{\text {tur }}+\mathrm{g}_{k}^{p u}\right)+\mathrm{K}_{\text {OM }}^{\text {bat }}\left(\mathrm{g}_{k}^{d i s}+\mathrm{g}_{k}^{c h}\right) \\
& C_{k}^{F}=P_{G T} \mathrm{~g}_{k}^{G T} \\
& C_{k}^{P E}=P_{2}^{k}\left|G_{k}-\mathrm{g}_{k}^{I I}-\mathrm{g}_{k}^{P V}-\mathrm{g}_{k}^{\mathrm{tur}}-\mathrm{g}_{k}^{d i s}+\mathrm{g}_{k}^{P U}+\mathrm{g}_{k}^{c h}-\mathrm{g}_{k}^{G T}\right|
\end{aligned}
$$

In the formula, $\mathrm{k}$ represents the time series, taking $15 \mathrm{~min}$ as a period, $\mathrm{k}=1,2,96 ; F_{k}^{V P P}$ is the net income of VPP in period $\mathrm{k} ; I_{k}^{S E}$ is the income of VPP in period $\mathrm{k}$; $P_{1}^{k}$ is TOU price of electricity sale price in period $\mathrm{k}$; $g_{k}^{I I} 、 g_{k}^{P V} 、 g_{k}^{G T} 、 g_{k}^{t u r} 、 g_{k}^{d i s} 、 g_{k}^{p u} 、 g_{k}^{c h}$ are respectively the power generation output of wind power, photovoltaic power, gas turbine power, pumped storage and energy storage battery and the charging power of pumped storage and energy storage battery in period $\mathrm{k} ; C_{k}^{O M}$ is the operation management cost of VPP in period $\mathrm{k}$ : $\mathrm{K}_{O M}^{W}$ 、

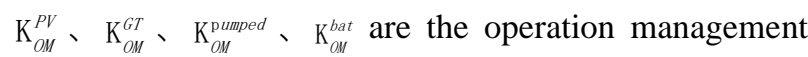
coefficient of wind power, photovoltaic, gas turbine, pumped storage and energy storage battery respectively; $C_{k}^{F}$ is the energy consumption cost of VPP in period k; $P_{G T}$ is the fuel cost of gas turbine per unit power generation; $C_{k}^{P E}$ is the penalty cost in period $\mathrm{k} ; P_{2}^{k}$ is the electricity purchase price of the time-of-use electricity price in period $\mathrm{k} ; G_{k}$. VPP declaration and contribution plan for period $\mathrm{k}$. 
The following formula is used to calculate the contribution of VPP to the distribution network's declaration plan:

$$
G_{k}=\left\{\begin{array}{l}
G_{\mathrm{s} k}+\eta g_{\max }^{G T} ; P_{2}^{\mathrm{k}}>P_{G T} \\
G_{\mathrm{s} k} ; P_{2}^{\mathrm{k}}<P_{G T}
\end{array}\right.
$$

Where: $G_{s k}$ is the uncontrollable DG plan output in period $\mathrm{k} ; \mathrm{n}$ is the output coefficient of gas turbine in period $\mathrm{k} ; g_{\max }^{G T}$ is the maximum output of gas turbine.

\subsection{Constraints}

(1) Power balance constraint:

$$
G_{\mathrm{k}}+g_{k}^{p u}+g_{k}^{c h}=g_{k}^{J I}+g_{k}^{P V}+g_{k}^{G T}+g_{k}^{t u r}+g_{k}^{d i s}+g_{k}^{d e v}
$$

$g_{k}^{d e r}$ represents the output deviation of VPP in period $\mathrm{k}$. .

(2) Controllable power supply constraints

$$
g_{\min }^{G T} \leq g_{k}^{G T} \leq g_{\max }^{G T}
$$

$g_{\max }^{G T}$ and $g_{\min }^{G T}$ are respectively upper and lower limits of gas turbine power.

(3) Pumped storage capacity constraints

$$
V_{\min }^{\text {up }} \leq V_{k}^{\text {up }} \leq V_{\max }^{\text {up }}
$$

$V_{\min }^{\text {up }}$ and $V_{\max }^{\text {up }}$ respectively represent the lower limit and upper limit of upper storage capacity.

(4) Energy storage battery energy and charge and discharge constraints

$$
\begin{gathered}
E_{\min }^{\mathrm{bat}} \leq E_{k}^{\mathrm{bat}} \leq E_{\max }^{\mathrm{bat}} \\
E_{k}^{\mathrm{bat}}=E_{k-1}^{\mathrm{bat}}+\Delta t\left(g_{\mathrm{k}}^{c h} n_{c}-g_{\mathrm{k}}^{d i s} / n_{d}\right) \\
u_{k}^{c h}+u_{k}^{d i s} \leq 1 \\
g_{\min }^{c h}<g_{k}^{c h}<g_{\max }^{c h} \\
g_{\min }^{d i s}<g_{k}^{d i s}<g_{\max }^{d i s}
\end{gathered}
$$

Where, $E_{\min }^{\text {bat }}$ and $E_{\max }^{\text {bat }}$ respectively represent the lower and upper limits of the capacity of the energy storage battery; $E_{k}^{\text {bat }}$ denotes the amount of energy storage battery in period $\mathrm{k} ; u_{k}^{c h}$ and $u_{k}^{d i s}$ represent state variables of charge and discharge respectively, with values of 0 or $1 ; g_{k}^{c h}$ and $g_{k}^{\text {dis }}$ respectively represent charging and discharging power in time period $\mathrm{k} ; n_{c}$ and $n_{d}$ represent charging and discharging efficiency respectively; $g_{\min }^{c h}$ and $g_{\max }^{c h}$ respectively represent the lower and upper limits of charging power; $g_{\min }^{d i s}$ and $g_{\max }^{d i s}$ represent lower limit and upper limit of discharge power respectively.

\section{Example analysis}

VPP is composed of $5220 \mathrm{MW}$ wind farms, 3 100MW photovoltaic power farms, 100MW gas turbine, 150MW pumped storage energy and 50MW energy storage battery. The relevant parameter information is as follows. The initial energy storage value of pumped storage energy is $180 \mathrm{MWh}$, and the initial energy storage value of energy storage battery is 50MWh. The electricity purchase prices in the flat section of peak and valley are 830,490 and 170 yuan/MWh respectively, and the electricity sale prices are 650, 380 and 130 yuan/MWh respectively. The wind power operation management coefficient is 29.6 yuan/MWh, and the photovoltaic operation management coefficient is 9.6 yuan/MWh. The lower limit of gas turbine output is $10 \mathrm{MW}$, the upper limit is $100 \mathrm{MW}$, the operation management coefficient is 120 yuan/MWh, the fuel cost coefficient is 168.6 yuan/MWh, the pumped storage operation management coefficient is $25.3 \mathrm{yuan} / \mathrm{MWh}$, the turbine power generation efficiency is 0.92 , the pump pumping efficiency is 0.81 , the pump output is $150 \mathrm{MW}$, The charge and discharge power of the energy storage battery is $50 \mathrm{MW}$, and the charge and discharge efficiency is 0.87 . The operation management coefficient is 84.3 yuan/MWh. The lower limit of the battery is $10 \mathrm{MWh}$, and the upper limit is 80MWh. Particle Swarm Optimization (PSO) algorithm is used to solve the economic optimal scheduling model of VPP. Parameters of particle swarm optimization algorithm are set as follows: population size is 50; The number of iterations is 300; Inertial weight factor $\mathrm{w}$ is 0.75 ; The learning factor was 1.5 .

The penalty cost of VPP represents the deviation of VPP output. When $\mathrm{n}$ is greater than 0.8 , the greater $\mathrm{n}$ is, the greater the penalty cost will be; when $n$ is less than 0.75 , the smaller $\mathrm{n}$ is, the greater the penalty cost will be. When $\mathrm{n}$ is 0.76 , the minimum penalty cost is 230,340 yuan, and the net benefit of VPP is 7689700 yuan. When $\mathrm{n}$ is 1, the gas turbine is at full power, and VPP has the best economic benefit. The simulation results show that VPP can adapt to the TOU price environment of distribution network by designing the operation strategy of controllable power supply, and choose the appropriate output coefficient to reduce the operation cost of VPP effectively, and obtain better economic benefits.

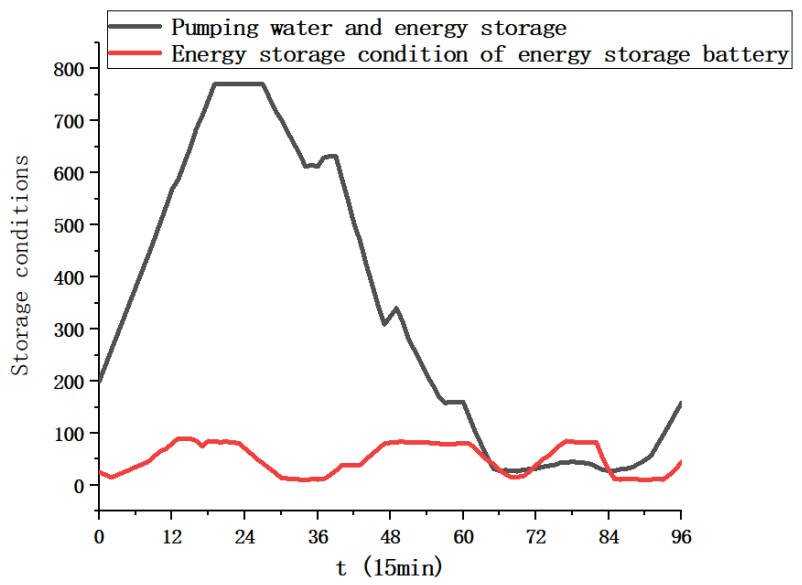

Fig. 1. Energy storage of pumped storage and energy storage batteries 


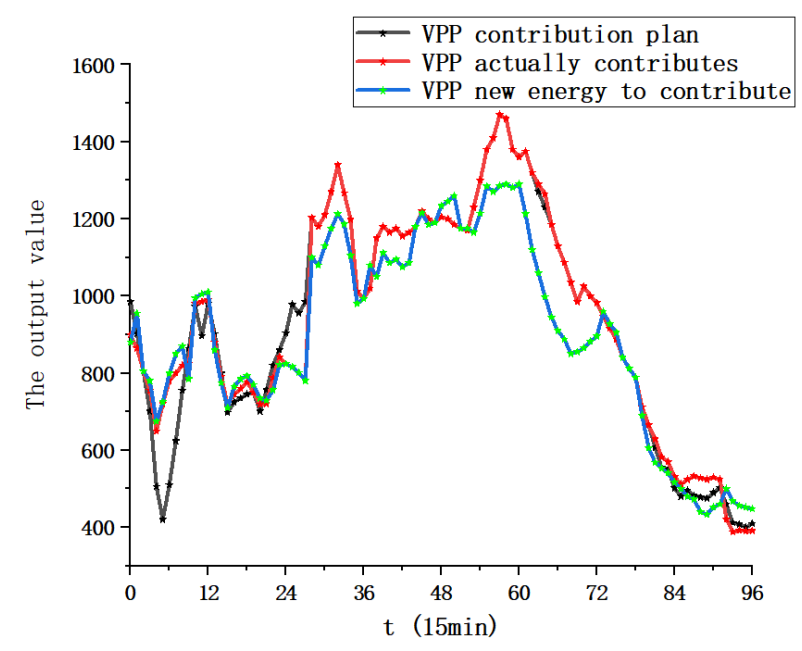

Fig. 2. The output

As shown in Fig. 1, pumped storage fills the valley and stores energy during periods 0 to 28 and 93 to 96 (TOU price valley period), compensates deviations during periods 29 to 92 (TOU price flat and peak period), and tries its best to participate in peak reduction during TOU price peak period. Energy storage batteries compensate deviations during the whole period.

Combined with Figure 2, the gas turbine stops during periods 0 to 28 and 93 to 96 . At this time, the VPP is powered by new energy, and only the energy storage battery runs, resulting in a large deviation in output. During the period of 29 to 92 , the gas turbine generates electricity. At this time, the VPP is powered by new energy and gas turbine. The pumped storage energy and the energy storage battery run together, with a small output deviation. According to calculation, when the TOU electricity price is in the valley, pumped storage energy filling in the valley and energy storage need to pay more penalty costs, so the net benefit is less. When the TOU electricity price is in the peak, the net benefit of VPP is higher. The calculated penalty cost paid by VPP to the distribution network is 249210 yuan. If VPP does not participate in peak clipping and valley filling, the net benefit of VPP is RMB 7,408,500. After participating in peak cutting and valley filling, the net benefit of VPP was RMB 7731200, the benefit of peak cutting and valley filling was RMB 322700, and the trough difference of distribution network was reduced by 100 MW. The simulation results verify the rationality of the model, which indicates that the construction of VPP can make DG better adapt to the environment of TOU electricity price and participate in the operation of the power grid. The TOU electricity price of the distribution network can effectively motivate VPP to participate in peak load cutting and valley filling to obtain greater benefits.

\section{Conclusion}

In this paper, a VPP operation strategy based on TOU electricity price is designed, and a VPP economic optimization scheduling model based on TOU electricity price is constructed with the goal of maximizing revenue in each time period. The output of each energy of VPP is obtained by particle swarm optimization algorithm. The simulation results verify the rationality of the model, which indicates that the construction of VPP can make DG better adapt to the environment of TOU electricity price and participate in the operation of the power grid, and the TOU electricity price can effectively motivate VPP to participate in peak load cutting and valley filling to obtain greater benefits.

\section{Acknowledgments}

This work was supported in part by National Natural Science Foundation of China under Grant 72001078 and Power System State Key Laboratory under Grant SKLD20M12.

\section{References}

1. WEI Zhinong, YU Shuang, SUN Guoqiang, et al. Concept and development of virtual power plant.J. Automation of Electric Power Systems, (2013).

2. LIU Jizhen, LI Mingyang, FANG Fang, et al. Review on virtual power plants.J. Proceedings of the CSEE, (2014).

3. YANG Jiajia, ZHAO Junhua, WEN Fushuan, et al.Development of bidding strategies for virtual power plants considering uncertain outputs from plug-in electric vehicles and wind generators.J. Automation of Electric Power Systems,(2014).

4. HE Qilin, AI Qian. Bidding strategy of electricity market including virtual power plant considering demand response under retail power market deregulation.J. Electric Power Construction, (2019).

5. WANG Xuanyuan, LIU Dunnan, LIU Zhen, et al. Operation mechanism and key technologies of virtual power plant under ubiquitous Internet of Things.J. Power System Technology,(2019).

6. LI Xudong, AI Xin, HU Junjie, et al. Three-stage combined peak regulation strategy for nuclearthermal-virtual power plant considering carbon trading mechanism.J. Power System Technology, (2019).

7. Wang J, Zhong H, Lai X, et al. Exploring key weather factors from analytical modeling toward improved solar power forecasting .J. IEEE Transactions on SmartGrid,(2019).

8. Dominguez R, Baringo L, Conejo A J. Optimal offering strategy for a concentrating solar power plant .J.Applied Energy,(2012).

9. Wang J , Zhong H, Xia Q,et al. Optimal jointdispatch of energy and reserve for CCHP-based microgrids .J. Iet Generation Transmission \& Distribution , (2017).

10. Liu G, Xu Y, Tomsovic K. Bidding strategy for microgrid in day -ahead market based on hybrid stochastic/robust optimization .J. IEEE Transactions on Smart Grid , (2015). 